\title{
Study on static characteristics of pneumatic muscles
}

\author{
Hu Wang ${ }^{1}$, Hongwei Yan ${ }^{1, a}$, Haodong Wang ${ }^{1}$, Zhong Yang ${ }^{1}$, Zhiguang $\mathrm{Ni}^{2}$, Zhe $\mathrm{Li}^{3}$ \\ ${ }^{1}$ College of Transportation, Ludong University, Yantai, China \\ ${ }^{2}$ School of Mechanical Engineering, University of Shanghai for Science and Technology, Shanghai, China \\ ${ }^{3}$ Department of Mechanical Engineering, Harbin Institute of Technology at Weihai, Weihai, China
}

\begin{abstract}
In this paper, the static characteristics of pneumatic muscles are studied by means of theoretical modelling, numerical simulation and experimental verification. Firstly, on the basis of ideal mathematical model, the static mathematical model of pneumatic muscle considering elasticity and friction of rubber is given. Based on the established mathematical model, a pneumatic muscle simulation model is established by using SIMULINK toolbox in MATLAB software environment, which includes model parameter assignment module, pneumatic muscle ideal module, elastic force simulation module, friction simulation module and so on. The influence of elastic force and frictional force of rubber layer on the output force of pneumatic muscle during pneumatic filling is studied by numerical simulation. Finally, a mechanical gripper test rig driven by pneumatic muscle is built. The experimental results show that pneumatic muscle actuator has certain flexible grasping characteristics. The research results provide a reference for the wide application of pneumatic muscle.
\end{abstract}

\section{Introduction}

With the development of manufacturing industry, actuators are required to have certain flexibility, adaptability and safety. However, the traditional motor and hydraulic drive methods can no longer meet the requirements, such as in the field of fragile assembly lines, human-oriented medical assistant robots and bionic service robots. Pneumatic muscles, as a new type of flexible actuator, have emerged as the times require. It can imitate the movements of human muscles. And the greatest feature of pneumatic muscles is their flexibility and adaptability, which are like the movements of human and animal limbs. Figure 1 shows the picture of FESTO's DMSP pneumatic muscle product.

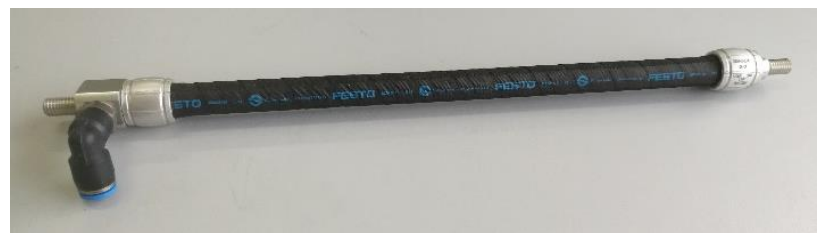

Figure 1. Picture of FESTO's DMSP pneumatic muscle

Because of the damping characteristics of the gas itself, it is beneficial to reduce the vibration of the actuator driven by pneumatic muscles during acceleration and deceleration. Using this characteristic, a threedegree-of-freedom flexible actuator with an elongated pneumatic artificial muscle was designed, which could be used as an actuator in bionic machinery such as agricultural robots and fruit and vegetable harvesting manipulators ${ }^{[1]}$. In addition, pneumatic muscle has the

\footnotetext{
a Corresponding author: yanhongwei123456@126.com
}

advantages of clean, low cost, easy installation and high energy/weight ratio. A new type of pneumatic muscle vest was put forward ${ }^{[2]}$. However, the inherent non-linear characteristics of gas, hysteresis, time-varying parameters, load sensitivity and the complexity of elastic and viscoelastic models of rubber materials make it difficult to control the output force and position of pneumatic muscles. C. J. Lin et al. studied the pressure/length hysteresis characteristics of pneumatic muscles and proposed a new model. The parameters of the model were identified by real code genetic algorithm ${ }^{[3]}$. LabVIEW and MATLAB system identification toolbox were used to identify the model parameters of the pneumatic position closed-loop control system. The data model of the complex system was obtained, which greatly saved the time of establishing the system mathematical model ${ }^{[4]}$. Because of the asymmetric hysteresis characteristic of pneumatic muscles, X. W. Yu et al. used the improved Jiles-Atherton model to describe the pneumatic muscles, and used the adaptive weighted particle swarm optimization to identify the parameters needed by the model, established the pneumatic muscles hysteresis model, and then realized the hysteresis compensation control of pneumatic muscles ${ }^{[5]}$. Aiming at the complicated modelling process of pneumatic artificial muscles, an experimental model identification method for pneumatic artificial muscles was proposed ${ }^{[6]}$. In addition, the complexity of real gas flow states makes accurate modeling of pneumatic muscles very complex and difficult. To avoid the difficulty of pneumatic muscle model and realize its position control, S. F. Wang et al. introduced a design method of pneumatic muscle 
controller. The phase leading part was used to reduce the residual vibration of the system, and the feed forward part was used to improve the position accuracy and tracking performance ${ }^{[7]}$. A cascade control strategy based on robust model method was proposed to realize the trajectory tracking control of a bionic joint driven by a single pneumatic artificial muscle ${ }^{[8]}$. To suppress buffeting of pneumatic artificial muscles, the discrete state equation was obtained by using PID control law instead of its mathematic model, and then the discrete Kalman recursive formula was incorporated into the discrete state equation. Then the Kalman PID control algorithm based on PID control parameters was proposed ${ }^{[9]}$. Aiming at the problems of small output force and insufficient range of motion of the existing single antagonistic configuration, a cascaded pneumatic muscle was designed to simulate human elbow joint ${ }^{[10]}$. Taking the physiological structure of human skeleton, joints and muscles as the design standard, and using the basic pneumatic muscle of fine-diameter wheat as the actuator, the bionic manipulator with good flexibility and light weight was designed, and the bionic movement of hand muscles was realized by using the bionic manipulator ${ }^{[11]}$. Through the analysis of previous studies, it can be found that the research on the driving characteristics of pneumatic muscle is not yet mature. Therefore, the static characteristics and driving characteristics of the pneumatic muscle are deeply studied in this paper.

\section{Mathematical model}

The main components of the pneumatic muscle are the inner rubber layer and the outer fiber weaving network. And its schematic diagram can be shown in Figure 2.

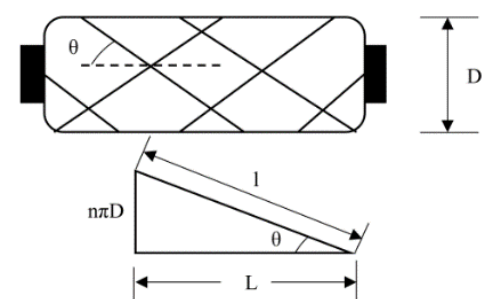

Figure 2. Structure diagram of pneumatic artificial muscle

The relationship between the relevant parameters in the pneumatic muscle structure can be described in the following way:

$$
\begin{gathered}
L=1 \cos \theta \\
n \pi D=1 \sin \theta \\
h=\frac{L_{N}-L}{L_{N}}
\end{gathered}
$$

$L_{N}, L$ and $D$ represent the initial length, actual length and outer diameter of pneumatic muscle respectively. $l, n, \theta$ and $h$ represent the length of single fiber, the number of filament winding, the braiding angle and shrinkage rate respectively.
Firstly, the ideal static mathematical model is established as:

$$
F_{\mathrm{i}}=\left(P_{\mathrm{t}}-P_{a}\right)\left(\frac{3 \pi \mathrm{D}_{\mathrm{N}}{ }^{2}}{4 \tan ^{2} \theta_{0}}(1-\mathrm{h})^{2}-\frac{\pi D_{N}{ }^{2}}{4 \sin ^{2} \theta_{0}}\right)
$$

$P_{t}$ and $P_{a}$ represent the absolute pressure and ambient atmospheric pressure.

And the elastic force of rubber is

$$
F_{s}=E_{\mathrm{x}} t_{r} \pi D_{0}\left\{\frac{(1-\mathrm{h})^{2}}{\tan ^{2} \theta_{0}}\left(1-\frac{\sin \theta_{0}}{\sin \theta}\right)+h \frac{\sin \theta}{\sin \theta_{0}}\right\}
$$

$E_{\mathrm{x}}$ and $t_{r}$ respectively represent the elastic modulus of pneumatic muscle and the thickness of rubber wall of pneumatic muscle.

The frictional force of pneumatic muscle can be described in the following way.

$$
F_{\mathrm{k}}=\mu\left\{\pi L_{N}(1-h)\left\{\left(\left(P_{\mathrm{t}}-P_{a}\right) D_{N}-2 E_{x} t_{r}\right) \frac{\sin \theta}{\sin \theta_{0}}+2 E_{\mathrm{x}} t_{r}\right\}\right\}
$$

In the formula, $F_{\mathrm{k}}$ and $\mu$ represent the internal friction of pneumatic muscles and the friction coefficient between rubber layer and manufacturing layer. The static mathematical model of pneumatic muscle is:

$$
F=\left(P_{\mathrm{t}}-P_{\mathrm{a}}\right)\left(\frac{3 \pi \mathrm{D}_{\mathrm{N}}^{2}}{4 \tan ^{2} \theta_{0}}(1-\mathrm{h})^{2}-\frac{\pi D_{N}^{2}}{4 \sin ^{2} \theta_{0}}\right)-F_{\mathrm{s}} \pm F_{\mathrm{k}}
$$

\section{Numerical simulation}

In order to study the relationship between the parameters of the pneumatic muscle mathematical model and the static characteristics of the pneumatic muscle, numerical simulation is needed. In this part, based on the mathematical model established in the previous section, the static simulation model of pneumatic muscle is established in MATLAB environment. On this basis, the simulation analysis is carried out.

\subsection{Establishment of simulation model}

The simulation model is established by using SIMULINK toolbox. It mainly includes assignment module, pneumatic muscle ideal module, elastic force simulation module, friction simulation module and so on. Figure 3 shows the ideal mathematical model simulation module, which is built based on the ideal mathematical model.

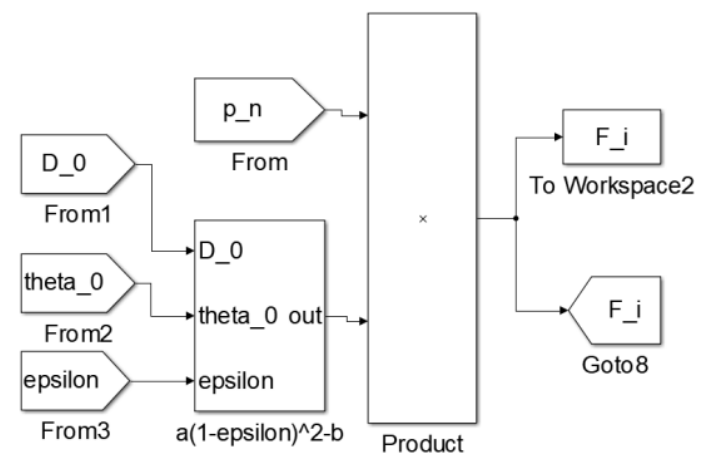

Figure 3. Simulation module of ideal mathematical model 
The elastic force mathematical model simulation module is based on the pneumatic muscle elastic force mathematical model, as shown in Figure 4. The simulation module of elastic force mathematical model plays a very important role in the whole simulation analysis.

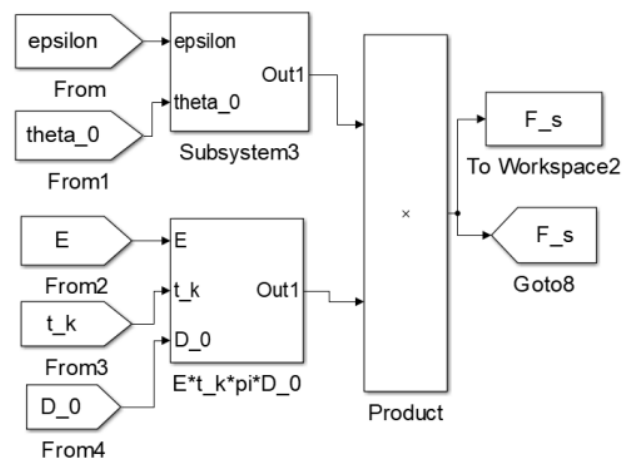

Figure 4. Elastic force simulation module

Figure 5 shows the simulation module of friction. In the process of inflation or exhaust, friction always obstruct the movement of pneumatic muscles.

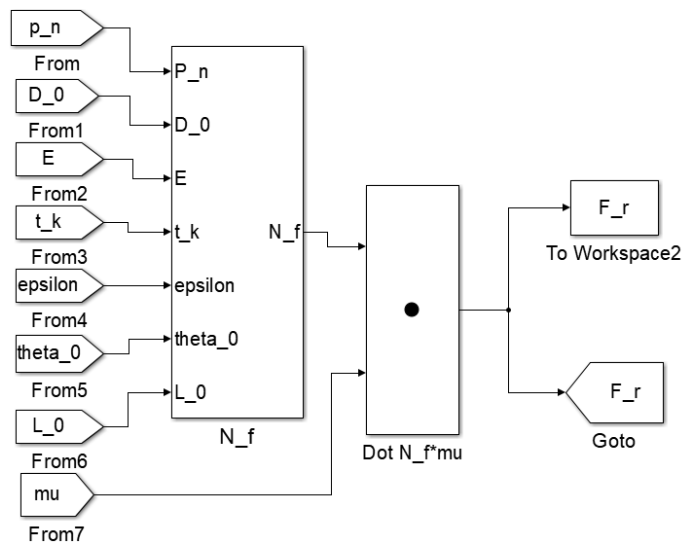

Figure 5. Friction simulation module

\subsection{Numerical simulation of static characteristics}

During the numerical simulation of pneumatic muscle static characteristics, the parameters used are as follows: the ambient pressure is $0.1 \mathrm{Mpa}$. The initial length, diameter and braiding angle of the pneumatic muscle are $0.25 \mathrm{~m}, 0.02 \mathrm{M}$ and 25 degrees respectively. The friction coefficient, rubber cylinder thickness and elastic modulus are $0.002,0.002 \mathrm{~m}, 1.3 \mathrm{Mpa}$ respectively. Firstly, the relationship between inflatable pressure and pneumatic muscle contraction force is analysed under the condition of equal length. At the same time, the ideal mathematical model is compared with the model considering elastic force and friction force. As you can see from Figure 6, the curve is straight. This is because the pneumatic muscle ideal mathematical model, ignoring a variety of nonlinear factors, is a linear mathematical model. No change in length direction is generated, no internal friction is involved. In addition, the friction between the rubber layer and the braided layer is very small, and it can be omitted from calculation. So, the two lines almost coincide.

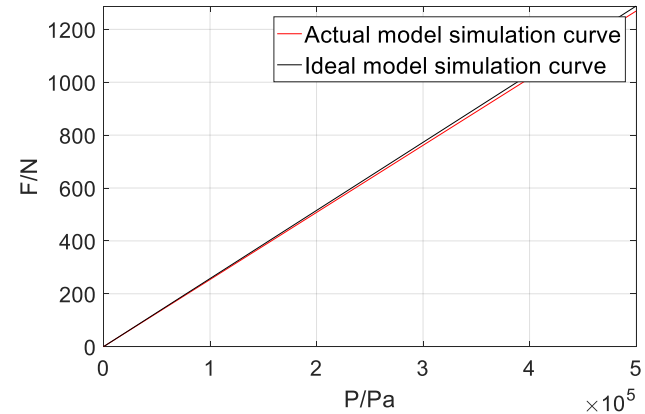

Figure 6. Contraction force VS inflatable pressure

The influence of shrinkage rate on output force under different inflatable pressure is shown in Figure 7. From the simulation curve, it can be seen that when the pneumatic muscle contraction rate is zero, the greater the filling pressure, the greater the pneumatic muscle output force. The influence of rubber elasticity on the static characteristics of pneumatic muscle has no direct relationship with the pneumatic pressure, only with the pneumatic muscle contraction rate.

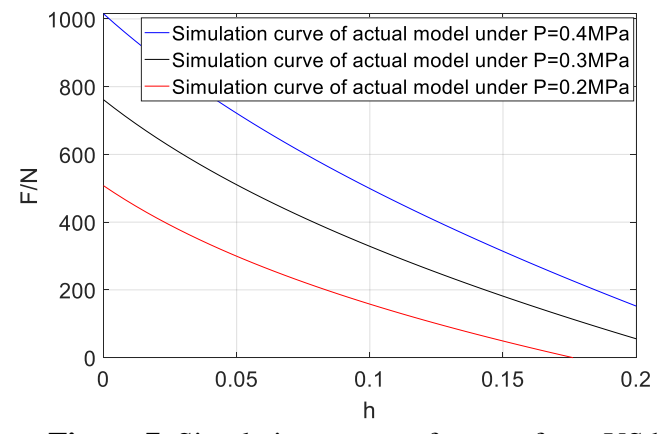

Figure 7. Simulation curves of output force VS h

Next, the relationship between the output force and the shrinkage rate of the pneumatic muscle is studied. The simulation curve is shown in the figure below.

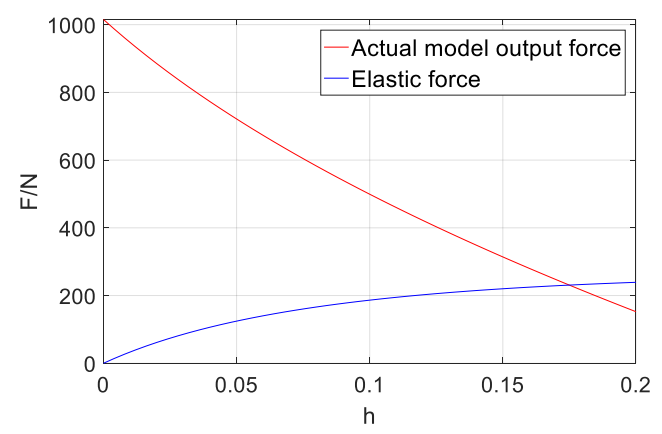

Figure 8. Relationship between $\mathrm{h}$ and Elastic force

In the simulation, the pressure $\mathrm{P}=0.4 \mathrm{MPa}$ is set and the shrinkage increases in a proportional function with time. Keeping the working pressure of pneumatic muscles constant, with the increase of contraction rate, the elastic force of rubber increases gradually, and the contractive force of pneumatic muscles decreases gradually. The elastic force has a countervailing effect on the output force of pneumatic muscles.

\section{Experiments and conclusion}


To verify the above theoretical analysis and simulation results, we set up an experimental platform in this section. Figure 9 shows the configuration of the test rig.

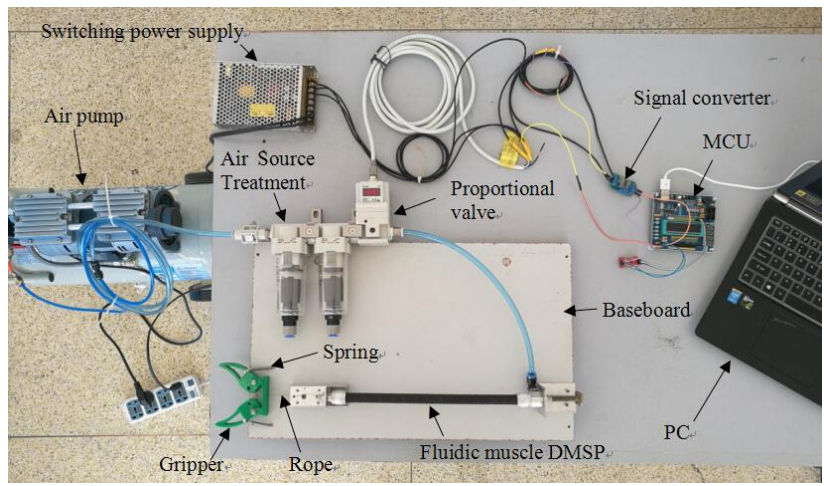

Figure 9. Photographs of the test rig

Experiments for grasping apple, peach, raw egg and grape are carried out. In the experiments, the control signal to the Electro-Pneumatic valve is $10.7 \mathrm{~mA} \mathrm{DC}$ current, which is obtained by trail method. The size and characteristics of the test sample are shown in Table 1.

Table 1. Dimensions and characteristics of test samples

\begin{tabular}{|c|c|c|}
\hline $\begin{array}{c}\text { Sample } \\
\text { name }\end{array}$ & $\begin{array}{c}\text { Size (in } \\
\text { diameter) }\end{array}$ & Characteristic \\
\hline Apple & $65 \mathrm{~mm}$ & Non-deformable \\
\hline Peach & $58 \mathrm{~mm}$ & $\begin{array}{c}\text { Slight deformation } \\
\text { allowable }\end{array}$ \\
\hline Raw egg & $41 \mathrm{~mm}$ & $\begin{array}{c}\text { Non-deformable and } \\
\text { fragile }\end{array}$ \\
\hline Grape & $22 \mathrm{~mm}$ & $\begin{array}{c}\text { Slight deformation } \\
\text { allowable }\end{array}$ \\
\hline
\end{tabular}

The experimental results show that the same control signal can be used to clamp objects with different sizes, which indicates that the mechanical clamping jaw driven by pneumatic muscle has certain flexibility. This is because with the increase of the contraction rate, the elastic force of rubber increases gradually, and the contraction force of pneumatic muscle decreases gradually. It shows that elastic force has an offset effect on the output force of pneumatic muscles. Further researches will be carried out from the following aspects: (1) Based on the conclusion of this paper, we further study the improvement of grasping efficiency. (2) New control algorithm will be explored to increase the system's adaptability furtherly.

\section{Acknowledgment}

This work is supported by the Training Foundation of Shandong Natural Science Foundation of China (NO. ZR2017PEE025), the Training Foundation of Shandong Natural Science Foundation of China (NO. ZR2017PEE016), the Talent Introduction Project of Ludong University (NO. LB2016001)

\section{References}

1. Y.W. Zhao, D.X. Geng, X.M. Liu, et al., Structure and deformation characteristics of 3-DOF pneumatic flexible actuator. Transactions of the Chinese society for agricultural machinery. Trans. Chinese. Soc. Agr., 48(9), 392-401 (2017).

2. C.J. Zhang, X.N. Li, A novel auxiliary vest powered by pneumatic muscle. Chinese. Hydraulic. Pneum., 12, 95-100 (2017).

3. C.J. Lin, C.R. Lin, S.K. Yu, et al., Hysteresis modelling and tracking control for a dual pneumatic artificial muscle system using Prandtl-Ishlinskii model. Mechatronics, 28, 35-45 (2015).

4. Z.L. Zhu, M.Y. Tang, L Kong, et al., On the cognition and analysis of pneumatic position system model based on LabVIEW and Matlab. J. Zunyi norm. coll., 18(3), 106-109 (2016).

5. X.W. Yu, H. Yang, L.N. Hao, Hysteresis modelling based on improved J-A model and compensation control for pneumatic muscle. Chinese. Hydraulic. Pneum., 7, 19-23 (2018).

6. L.N. Sun, Q. Bi, Empirical model based study in position control of pneumatic artificial muscle. Mach. tool hydraulic., 46(5), 84-86, 142 (2018).

7. S.F. Wang, K. Sato, High-precision motion of a stage with pneumatic artificial muscle. Precis. Eng., 43, 448-461 (2016).

8. Y. Wang, Q. Zhang, X.H. Xiao, et al., Trajectory Tracking Control of the Bionic Joint Actuated by Pneumatic Artificial Muscle Based on Robust Modelling. Robot, 38(2), 248-256 (2016).

9. H. Yang, L.N. Hao, Y. Chen, B.C. Xue, Kalman-PID control for chattering phenomena of bionic elbow joint actuated by pneumatic artificial muscles. Control theory appl., 34(4), 477-482 (2017).

10. R.B. Wang, B. Zhang, G.Y. Shen, et al., Modeling and fuzzy control of humanoid elbow driven by cascaded pneumatic muscles. Robot, 39(4), 474-480 (2017).

11. Z.J. Nan, Y. Yang, SUZUMORI Koichi, et al., Development of a bionic hand actuated by thin McKibben pneumatic artificial muscle. Robot, 40(3), 321-328 (2018). 